\title{
Selección de sitios de anidación de Lepidochelys olivacea (Testudines: Cheloniidae) en el Pacífico Sur de Costa Rica
}

\author{
Andrés Ávila-Aguilar \\ Escuela de Biología, Universidad de Costa Rica, San Pedro, Costa Rica; andres.avila97@gmail.com
}

Recibido 11-VIII-2014. Corregido 20-XI-2014. Aceptado 22-XII-2014.

\begin{abstract}
Nest-site selection of Lepidochelys olivacea (Testudines: Cheloniidae) in the South Pacific region of Costa Rica. In sea turtles, nest-site selection plays a key role in the survival of the offspring, with a direct influence on the sex of the hatchlings, incubation period, embryonic mortality, and hatchling size. Nest-site choice has been studied for most sea turtle species, but the information for Lepidochelys olivacea, the most abundant sea turtle species, is scarce. The present work explores the spatial nesting patterns of $L$. olivacea and seeks to determine whether this species employs a scatter nesting strategy or chooses to clump its nests based on a specific spatial preference. Night patrols were conducted between July and September 2011 at the beaches of Piro and Pejeperro, located in the Osa Peninsula, in the South Pacific region of Costa Rica. Nest-site location was evaluated by measuring the distance from the tide line to the nest, distance from nest to vegetation, and by noting beach sector according to beach-marker setup. In total, 84 nesting turtles were observed. Sea turtles in both beaches showed a strong preference to locate their nests as far from the tide line as possible, crawling longer distances when presented with wider stretches of beach. Turtles nesting at Piro beach also favored areas that were close to the river mouth, while turtles at Pejeperro preferred to nest near an estuary. It seems that the main driving force that influences L. olivacea to choose a nest site is to protect the eggs from tidal inundation, even if this means a higher predation risk from nesting too close to the vegetation, in addition to a longer trip to the ocean for both the female adult and the hatchlings. Nests near rivers and estuaries may have higher hatching success, which could prompt females to return and select these areas due to imprinting or philopatry. Future research focused on recapture could provide valuable information on individual behavior, and prove whether or not L. olivacea shows strong nest-site fidelity and repeatability of nesting preferences at the individual level. Rev. Biol. Trop. 63 (Suppl. 1): 375-381. Epub 2015 April 01.
\end{abstract}

Key words: Nest-site selection, Lepidochelys olivacea, Osa Peninsula, philopatry, imprinting.

La elección del sitio de anidación para tortugas marinas es un determinante crítico para el desarrollo y sobrevivencia de la progenie. La posición del nido y las condiciones ambientales dentro del mismo influyen directamente en la determinación del sexo (Mrosovsky \& Yntema, 1980), el periodo de incubación (Ackerman, 1997), la mortalidad embriónica (Mortimer, 1990) y el tamaño de los neonatos (Packard \& Packard, 1994).

Para la tortuga cabezona Caretta caretta se ha evaluado la ubicación del nido y los posibles mecanismos y consecuencias que influyen en la ecología de dicha selección, (Williams-Walls et al., 1983; Hays \& Speakman, 1993; Garmestani, Percival, Portier \& Rice, 2000), también para la tortuga verde Chelonia mydas (Bjorndal \& Bolten, 1992; Weishampel, Bagley, Ehrhart \& Rodenbeck, 2003), la tortuga baula Dermochelys coriacea (Mrosovsky, 1983; Eckert, 1987; Nordmoe et al., 2004), y la tortuga carey Eretmochelys imbricata (Horrocks \& Scott, 1991). Existe poca información en cuanto a la selección y distribución de sitios de anidación de la tortuga lora Lepidochelys olivacea, como el estudio realizado por López-Castro, Carmona y Nichols (2004) en la región de Baja California, la cual marca el límite norte de la zona 
de anidación de esta especie. Hasta el momento no existen otros trabajos que hayan abordado este tema en otras regiones del trópico.

Lepidochelys olivacea es la especie de tortuga marina más abundante que existe, debido en parte al uso de sitios de anidación masiva (arribadas) en Costa Rica, México y la India (Pritchard, 1997). La anidación simultánea de miles de hembras reproductivas en las playas de arribada puede haber interferido en los esfuerzos por dilucidar patrones claros en cuanto a la selección del sitio de anidación. Por lo tanto, para estudiar este aspecto se requiere de una playa de anidación que no presente el fenómeno de arribada, pero que al mismo tiempo sea visitada por un número suficiente de individuos que permita inferir posibles tendencias en la población.

Las playas de la península de Osa sirven como zona de anidación para cuatro especies de tortugas marinas: L. olivacea, C. mydas agassizii, E. imbricata y D. coriacea. El presente trabajo tiene como objetivo determinar si las hembras de L. olivacea seleccionan consistentemente el sitio de anidación según un patrón o preferencia espacial determinada, o si por el contrario distribuyen los nidos de forma aleatoria en las playas de Piro y Pejeperro; dos sitios que cuentan con las condiciones propicias para inferir patrones poblacionales en cuanto a la selección y localización de nidos. Esta investigación se enfocó únicamente en la actividad anidatoria de L. olivacea.

\section{MATERIALES Y MÉTODOS}

Área de estudio: las playas Piro y Pejeperro están ubicadas en el sector sur de la Península de Osa, Costa Rica, entre los $8^{\circ} 24^{\prime}-8^{\circ} 23^{\prime}$ $\mathrm{N}-83^{\circ} 23^{\prime}-83^{\circ} 19^{\prime} \mathrm{W}$. Ambas playas tienen una longitud aproximada de $9 \mathrm{~km}$. La precipitación media anual de esta zona es de $5500 \mathrm{~mm}$, y la temperatura media anual es $27^{\circ} \mathrm{C}$ (SánchezAzofeifa, Rivard, Calvo \& Moorthy, 2002). Estas playas se ubican en una zona costera remota y hasta el momento relativamente poco intervenida, con presencia de vegetación propia del bosque tropical lluvioso. Las playas están marcadas en sectores rotulados cada $100 \mathrm{~m}$.

En playa Piro, la arena de los primeros sectores es de grano más grueso y en general presenta una consistencia más pedregosa que el resto de la playa. La desembocadura del río Piro se ubica cerca de los sectores nueve y diez. En cuanto a playa Pejeperro, la zona intermareal de los sectores uno a cinco está ocupada por rocas de gran tamaño, lo cual obstaculiza la llegada de las tortugas e impide la anidación en estos sectores. En el extremo noroeste de la playa se encuentra el estero o laguna Pejeperro, la cual durante la época lluviosa desemboca al mar en las cercanías del sector 45 .

Muestreo: Se realizaron patrullajes nocturnos en ambas playas entre julio y setiembre del 2011. Cada tortuga encontrada se marcó con etiquetas metálicas en ambas aletas frontales y con un Passive Integrated Transponder (PIT tag). Para cada tortuga se registró la fecha y hora del evento, la ubicación del nido utilizando el número de sector de la playa rotulados cada $100 \mathrm{~m}$, la distancia lineal de la línea de marea al nido (m), la distancia lineal del nido a la vegetación $(\mathrm{m})$, la condición del nido (in situ, salida falsa o depredado), la longitud curva del caparazón (cm), el ancho de la huella $(\mathrm{cm})$, el número de etiqueta de la aleta izquierda, el número de etiqueta de la aleta derecha, el número de PIT tag y comentarios adicionales.

Análisis de datos: Los datos fueron editados mediante la transformación estadística de Box-Cox para que las regresiones lineales se ajustaran al principio de normalidad. Los análisis fueron realizados con los programas JMP y STATISTICA.

\section{RESULTADOS}

Se registraron 84 eventos de anidación: 32 en playa Piro y 52 en playa Pejeperro. En ambas playas se detectó una fuerte relación lineal entre la distancia recorrida por las tortugas desde el agua hasta el sitio de anidación, y el ancho total de playa disponible (Playa Piro 
(Fig. 1): $\mathrm{F}_{1,31}=71.58, \mathrm{p}<0.0001, \mathrm{r}^{2}=0.70$; Playa Pejeperro (Fig. 2): $\left(\mathrm{F}_{1,51}=240.94, \mathrm{p}<0.0001, \mathrm{r}^{2}\right.$ $=0.82$ ).

En los distintos sectores de playa Piro, los eventos de anidación no se distribuyeron de forma homogénea $\left(\mathrm{X}^{2}=34.27, \mathrm{p}=0.017\right.$, g.l.=19), registrándose una marcada preferencia de las tortugas por los sectores nueve y diez cercanos a la desembocadura del río Piro (Fig. 3).

Los eventos de anidación en playa Pejeperro tampoco se distribuyeron equitativamente

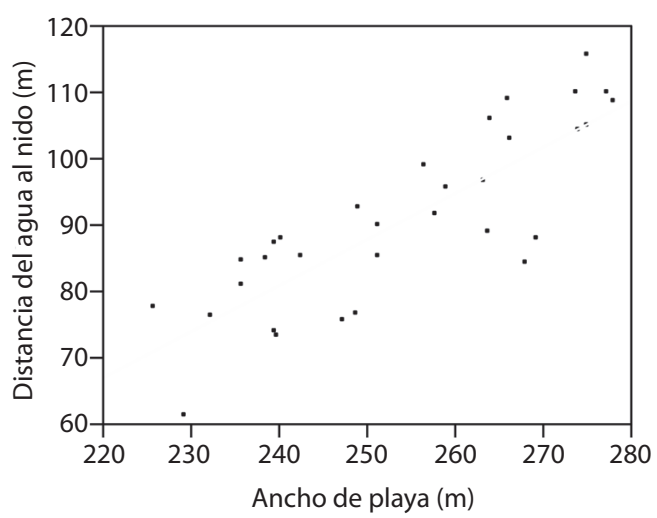

Fig. 1. Relación entre la distancia recorrida por L. olivacea del agua al nido, y el ancho de playa disponible en playa Piro.

Fig. 1. Relationship between the distance traveled by $L$. olivacea from the water line to the nest, and the beach width available at the moment of nesting at Piro beach.
$\left(\mathrm{X}^{2}=63.32, \mathrm{p}=0.030, \mathrm{~g} .1 .=44\right)$. En este caso, la preferencia se concentró en la segunda mitad de la playa, a partir del sector 23 y cerca de la zona de estero (Fig. 4).

En cuanto a las frecuencias de salidas falsas (tortugas que llegaron a la playa pero decidieron no anidar en ese momento), en los distintos sectores de Playa Piro se distribuyeron de forma homogénea (Fig. 5: $\mathrm{X}^{2}=15.54$, $\mathrm{p}=0.69$, g.l. $=19$ ), siendo similar a lo registrado en playa Pejeperro (Fig. 6: $\mathrm{X}^{2}=55.56, \mathrm{p}=0.11$, g.l.=44). Estos resultados descartan la hipótesis

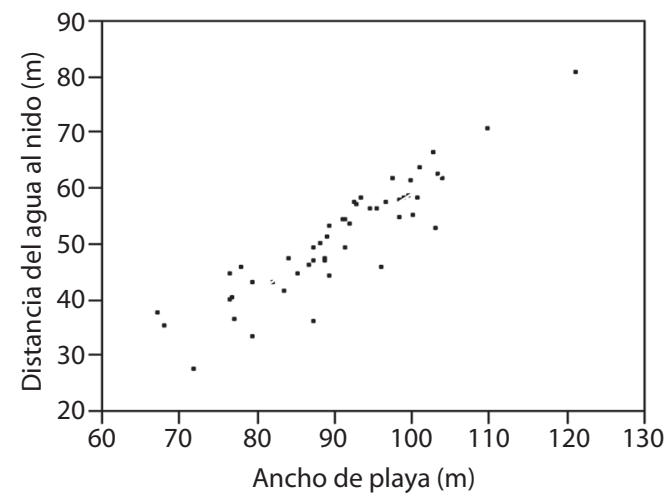

Fig. 2. Relación entre la distancia recorrida por L. olivacea del agua al nido, y el ancho de playa disponible en playa Pejeperro.

Fig. 2. Relationship between the distance traveled by $L$. olivacea from the water line to the nest, and the beach width available at the moment of nesting at Pejeperro beach.

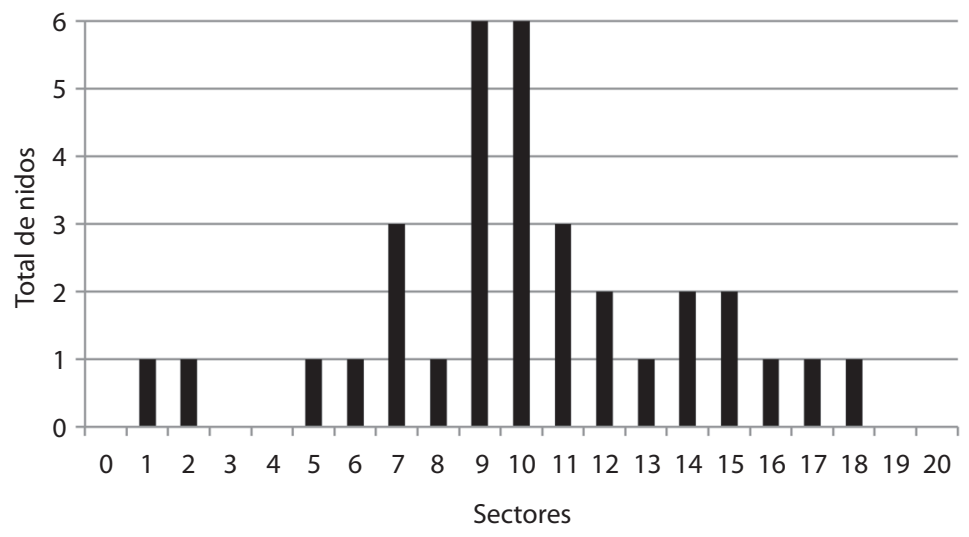

Fig. 3. Frecuencias de eventos de anidación para cada uno de los sectores de playa Piro.

Fig. 3. Nesting frequencies observed along the sectors of Piro beach. 


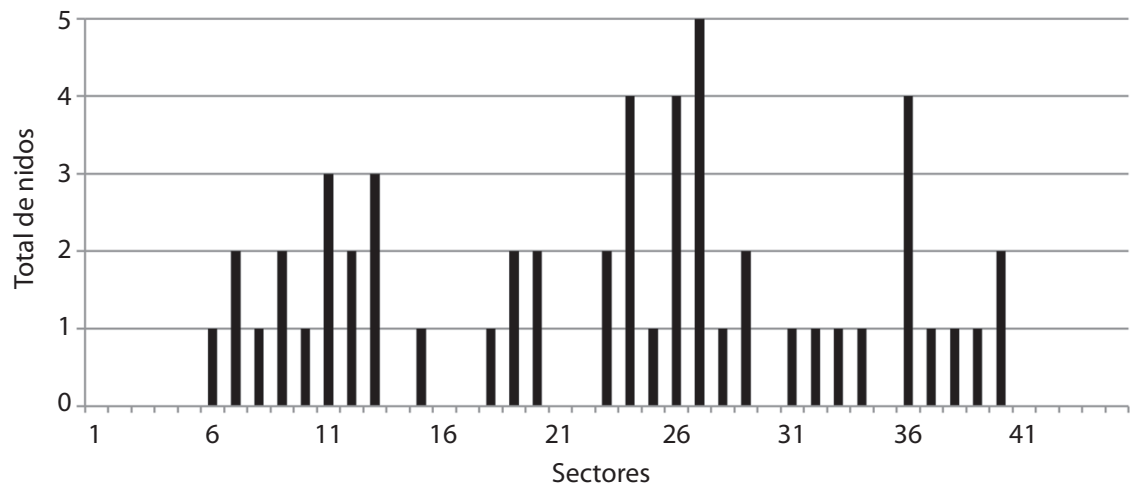

Fig. 4. Frecuencias de eventos de anidación para cada uno de los sectores de playa Pejeperro.

Fig. 4. Nesting frequencies observed along the sectors of Pejeperro beach.

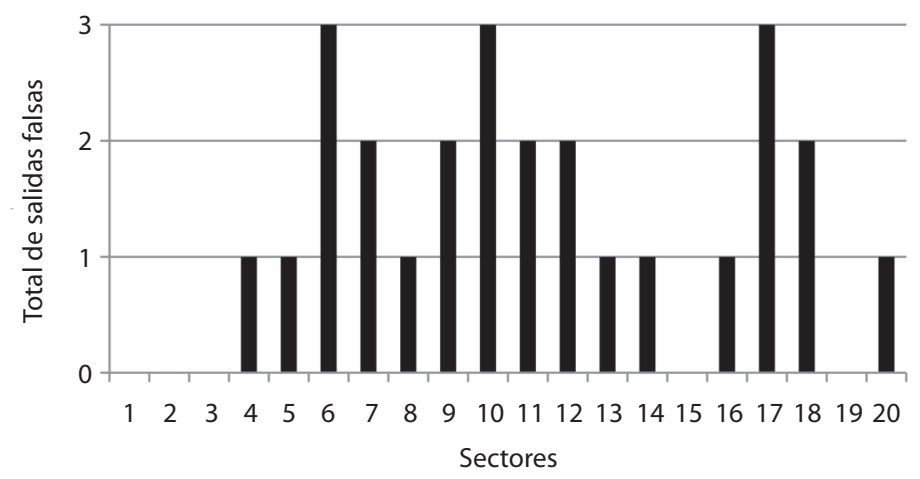

Fig. 5. Frecuencias de salidas falsas para cada uno de los sectores de playa Piro.

Fig. 5. False-crawl frequencies observed along the sectors of Piro beach.

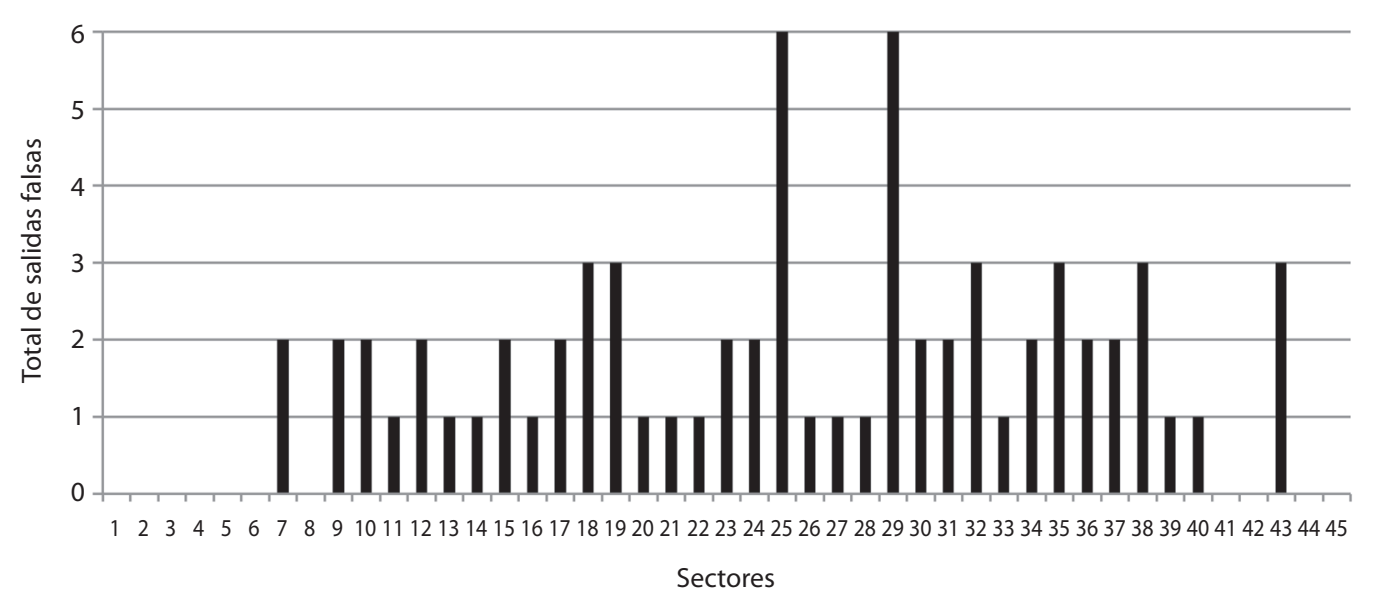

Fig. 6. Frecuencias de salidas falsas para cada uno de los sectores de playa Pejeperro.

Fig. 6. False-crawl frequencies observed along the sectors of Pejeperro beach. 
inicial de que la mayor cantidad de salidas falsas se asociaría a sectores con menor cantidad de nidos.

\section{DISCUSIÓN}

Para las playas de Piro y Pejeperro se confirma que L. olivacea selecciona los sitios de anidación de acuerdo a un patrón de preferencia establecido, el cual se basa en el ancho de playa disponible al momento de anidar. La fuerte relación lineal observada entre esta variable y la distancia del agua al nido muestra la marcada preferencia de L. olivacea por ubicar consistentemente sus nidos lo más lejos posible de la línea de marea. Esto contrasta significativamente con la estrategia empleada por D. coriacea en Guyana Francesa (Mrosovsky, 1983) e Islas Vírgenes (Eckert, 1987), la cual prefiere distribuir sus nidos de forma aleatoria a lo ancho de la playa, sin un patrón definido y en muchas ocasiones debajo de la línea de marea alta, quedando expuestos al oleaje y a la pérdida inevitable de embriones. Lo que podría considerarse una pobre estrategia de selección de sitios de anidación, para $D$. coriacea sería una estrategia de dispersión de nidos al azar con el fin de maximizar la sobrevivencia de la progenie en zonas de anidamiento con éxito de eclosión impredecibles (Nordmoe et al., 2004).

Para L. olivacea en la Península de Osa, el hecho de ubicar los nidos lo más alejado de la línea de marea alta posible para protegerlos de la marea parece incluso tener más relevancia que el riesgo de depredación asociado a anidar en zonas cercanas a la vegetación. Al respecto, la preferencia de esta especie por sitios de anidación alejados de la línea de marea es similar a lo observado por Hays y Speakman (1993) en poblaciones de $C$. caretta en Grecia, y por Horrocks y Scott (1991) en el caso de E. imbricata en Barbados, mientras que la estrategia de $D$. coriacea ha sido registrada también para $C$. mydas en las playas de Tortuguero, Costa Rica (Bjorndal \& Bolten, 1992).

Es importante indicar que en las playas estudiadas, L. olivacea también mostró preferencia por anidar en sitios cercanos a desembocaduras de ríos (sectores nueve y diez en playa Piro) y esteros (segunda mitad de playa Pejeperro). Esta preferencia ha sido registrada también por López-Castro et al. (2004), sin embargo no se han definido las condiciones específicas que las tortugas marinas "evalúan" in situ para construir el nido. Mortimer (1990) examinó muchas propiedades físicas y químicas de la arena para dilucidar el mecanismo de selección en $C$. mydas, incluyendo la distribución del tamaño de las partículas de arena, textura, porosidad, contenido de agua, conductividad eléctrica, $\mathrm{pH}$, salinidad y potencial hídrico, y encontró que la construcción de nidos en arenas de grano grueso e irregular presenta una mayor dificultad para las tortugas y está correlacionada con un mayor índice de mortalidad de neonatos, debido a que los nidos construidos tienden a colapsar cuando los huevos eclosionan. Esto podría explicar lo observado en las playas de Piro y Pejeperro, donde existe una ausencia conspicua de nidos en sectores de la playa donde la arena es más gruesa y pedregosa.

Los resultados también mostraron que las salidas falsas se distribuyeron de forma homogénea a lo largo de la playa, contrario a lo esperado en cuanto a que sectores con menor frecuencia de anidación posiblemente registrarían un mayor número de salidas falsas. Esto podría deberse a la variación de las condiciones ambientales de un mismo sitio en periodos distintos, y la influencia de estas variaciones en la decisión final de anidar o no; es decir, un sitio puede ser apto y deseable para anidar en un momento, y dejar de serlo en otro.

Si los sectores cercanos a desembocaduras de ríos y esteros están asociados a un mayor éxito de eclosión, es posible que las tortugas nacidas ahí estén regresando a esos mismos sitios en su etapa adulta y seleccionándolos por impronta o filopatría, lo cual también ayudaría a explicar la mayor concentración de nidos en estas zonas tan específicas. A esto se debe sumar la posibilidad de repetibilidad en cuanto a la selección del sitio de anidación, en el cual una hembra muestra de forma consistente las mismas preferencias para anidar en 
visitas sucesivas a la playa. El fenómeno de repetibilidad ha sido documentado principalmente para E. imbricata (Kamel \& Mrosovsky, 2005, 2006), y aunque algunas observaciones de campo sugieren que $L$. olivacea también presenta este tipo de comportamiento, la información al respecto es mayoritariamente anecdótica. En el presente estudio no fue posible evaluar repetibilidad debido a que el número de recapturas durante el periodo de muestreo fue muy bajo; esto ofrece una oportunidad para futuras investigaciones que se enfoquen en las preferencias de anidación de L. olivacea a nivel de individuo, lo cual constituiría una contribución importante al conocimiento de la historia natural de esta especie.

\section{AGRADECIMIENTOS}

A Gerardo Chávez por su orientación, sugerencias y material bibliográfico recomendado. A Guido Saborío y Osa Conservation por el apoyo logístico brindado. A Manuel Sánchez, Phoebe Edge, Daniella Ulloa y el equipo de voluntarios de Osa Conservation y Frontier UK por su asistencia con la recolección de datos en el campo. Al Proyecto Consolidación de Áreas Marinas Protegidas del Sistema Nacional de Áreas de Conservación (SINAC), Programa de Naciones Unidas para el Desarrollo (PNUD) y el Global Environment Facility (GEF) por su colaboración en la publicación.

\section{RESUMEN}

La selección del sitio de anidación en tortugas marinas es determinante para la sobrevivencia de la progenie, sin embargo para Lepidochelys olivacea existe poca información al respecto. Este trabajo se enfocó en determinar si L. olivacea selecciona sus sitios de anidación de acuerdo a un patrón o preferencia espacial determinada, o si por el contrario distribuye sus nidos aleatoriamente en la playa. La recolección de datos se realizó entre julio y setiembre del 2011, en las playas de Piro y Pejeperro, Península de Osa, Costa Rica. La ubicación del nido se evaluó midiendo las distancias entre la línea de marea y el nido, entre el nido y la vegetación, y determinando la posición del nido con respecto a los sectores marcados cada $100 \mathrm{~m}$ en la playa. En ambas playas, las tortugas mostraron una marcada preferencia por anidar lo más lejos posible de la línea de marea, lo cual protege a sus nidos de inundación pero los expone a un mayor riesgo de depredación. Además, en playa Piro se detectó una mayor concentración de nidos cerca de la desembocadura del río, y en las cercanías de un estero en el caso de Pejeperro. Si estas zonas están asociadas a un mayor éxito de eclosión, es posible que las tortugas estén regresando y seleccionado esos sitios por impronta o filopatría. Futuras investigaciones deberán enfocarse en determinar si en eventos de anidación sucesivos, L. olivacea muestra repetibilidad y mantiene sus preferencias de anidación a nivel de individuo.

Palabras clave: Selección del sitio de anidación, Lepidochelys olivacea, Península de Osa, filopatría, impronta.

\section{REFERENCIAS}

Ackerman, R. A. (1997). The nest environment and the embryonic development of sea turtles. In P. L. Lutz \& J. A. Musick (Eds.), The Biology of Sea Turtles (pp. 83-106). Florida: CRC Press.

Bjorndal, K. A., \& Bolten, A. B. (1992). Spatial distribution of green turtle (Chelonia mydas) nests at Tortuguero, Costa Rica. Copeia, 1992, 45-53.

Eckert, K. L. (1987). Environmental unpredictability and leatherback sea turtle (Dermochelys coriacea) nest loss. Herpetologica, 43, 315-323.

Garmestani, A. S., Percival, H. F., Portier, K. M., \& Rice, K. G. (2000). Nest-site selection by the loggerhead sea turtle in Florida's Ten Thousand Islands. Journal of Herpetology, 34, 504-510.

Hays, G. C., \& Speakman, J. R. (1993). Nest placement by loggerhead turtles, Caretta caretta. Animal Behaviour, 45, 47-53.

Horrocks, J. A., \& Scott, N. M. (1991). Nest site location and nest success in the hawksbill turtle Eretmochelys imbricata in Barbados, West Indies. Marine Ecology Progress Series, 69, 1-8.

Kamel, S. J., \& Mrosovky, N. (2005). Repeatibility of nesting preferences in the hawksbill sea turtle, Eretmochelys imbricata, and their fitness consequences. Animal Behaviour, 70, 819-828.

Kamel, S. J., \& Mrosovky N. (2006). Inter-seasonal maintenance of individual nest site preferences in hawksbill sea turtles. Ecology, 87, 2947-2952.

López-Castro, M. C., Carmona, R., \& Nichols, W. J. (2004). Nesting characteristics of the olive ridley turtle (Lepidochelys olivacea) in Cabo Pulmo, southern Baja California. Marine Biology, 145, 811-820.

Mortimer, J. A. (1990). The influence of beach sand characteristics on the nesting behavior and clutch survival of green turtles (Chelonia mydas). Copeia, 1990, 802-817. 
Mrosovsky, N., \& Yntema, C. L. (1980). Temperature dependence of sexual differentiation in sea turtles: implications for conservation practices. Biological Conservation, 18, 271-280.

Mrosovsky, N. (1983). Ecology and nest-site selection of leatherback turtles Dermochelys coriacea. Biological Conservation, 26, 47-56.

Nordmoe, E. D., Sieg, A. E., Sotherland, P. R., Spotila, J. R., Paladino, F. V., \& Reina, R. D. (2004). Nest site fidelity of leatherbacks at Playa Grande, Costa Rica. Animal Behaviour, 68, 387-394.

Packard, G. C., \& Packard, M. J. (1994). The physiological ecology of reptilian eggs and embryos. In C. Gans \& R. B. Huey (Eds.), Biology of the reptilia 16. Ecology B: defense and life history (pp. 523-605). Michigan, USA: Branta Books.
Pritchard, P. C. H. (1997). Evolution, phylogeny, and current status. In P. L. Lutz \& J. A. Musick (Eds.), The biology of sea turtles (pp. 2-24). Florida, USA: CRC Press.

Sánchez-Azofeifa, G. A., Rivard, B., Calvo, J., \& Moorthy, I. (2002). Dynamics of tropical deforestation around national parks: remote sensing of forest change on the Osa Peninsula of Costa Rica. Mountain Research and Development, 22, 352-358.

Weishampel, J. F., Bagley, D. A., Ehrhart, L. M., \& Rodenbeck, B. (2003). Spatiotemporal patterns of annual sea turtle nesting behaviors along an East Central Florida beach. Biological Conservation, 110, 295-303.

Williams-Walls, N., O'Hara, J., Gallagher, R. M., Worth, D. F., Peery, B. D., \& Wilcox, J. R. (1983). Spatial and temporal trends of sea turtle nesting on Hutchinson Island, Florida, 1971-1979. Bulletin of Marine Science, 33, 55-66. 
